\title{
Digital file format does not influence the radiographic diagnosis of vertical root fracture
}

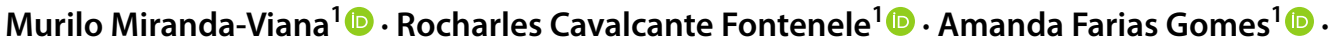

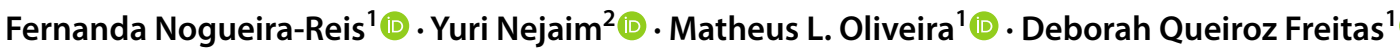

Received: 12 July 2021 / Accepted: 28 September 2021 / Published online: 9 October 2021

(c) The Author(s), under exclusive licence to Japanese Society for Oral and Maxillofacial Radiology and Springer Nature Singapore Pte Ltd. 2021

\begin{abstract}
Objective To evaluate the influence of the file format of digital periapical radiographs on the diagnosis of vertical root fracture (VRF).

Study design Periapical radiographic images of 34 single-rooted teeth-19 with VRF, and 15 without VRF were acquired using two digital systems-Digora Toto, and Digora Optime, and exported into four different file formats-TIFF, BMP, PNG, and JPEG, totaling 272 radiographs. The radiographs were assessed by five examiners for the detection of VRF, using a 5-point scale (1-definitely absent; 2-probably absent; 3-uncertain; 4-probably present; 5-definitely present). Diagnostic values of area under the ROC curve, specificity, and sensitivity for the diagnosis of VRF were calculated. The results were compared by two-way Analysis of Variance with post hoc Tukey's test. The intra- and inter-examiner agreements were measured by the Kappa test. The significance level was set at 5\% for all analyses.

Results The values of intra-examiner agreement varied from moderate (0.56) to almost perfect (0.81), while the values of inter-examiner agreement varied from fair $(0.29)$ to moderate $(0.60)$. The image file format did not influence the diagnostic values for VRF for any of the radiographic systems tested $(p>0.05)$. Digora Toto had significantly greater values of area under the ROC curve than Digora Optime for all file formats $(p=0.001)$.

Conclusion The image file format of periapical radiographs does not influence the diagnosis of VRF, regardless of the digital radiography system.
\end{abstract}

Keywords Dental digital radiography $\cdot$ Diagnostic imaging $\cdot$ Tooth fractures

\section{Introduction}

Periapical radiographs are commonly used to establish the diagnosis and treatment planning for several dental conditions (e.g., root fractures), as it allows a satisfactory assessment of the crown, root, and surrounding bone [1]. Among the diagnostic tasks, the diagnosis of vertical root fractures (VRFs), based on clinical and radiographic assessments, is considered challenging. However, an early diagnosis,

Rocharles Cavalcante Fontenele

rocharlesf@gmail.com

1 Oral Radiology Area, Piracicaba Dental School, University of Campinas, Avenida Limeira, 901, Piracicaba, São Paulo 13414-903, Brazil

2 Oral Radiology Area, Dental School, Federal University of Mato Grosso do Sul, Campo Grande, MS, Brazil whether clinical or radiographic, is essential for a better prognosis of VRF [2].

Vertical root fractures are often not visible on clinical examination, do not have a favorable prognosis, being one of the most common reasons for the extraction of endodontically treated teeth [3]. When radiographically assessed, vertical fractures can present as an incomplete or complete radiolucent line that extends along the longitudinal axis of the tooth. It can even cause complete separation of the fragments, but in most cases, factors, such as fracture width and location, can complicate the diagnosis [4]. This radiographic assessment differs from the horizontal type that often originates from great traumatic forces and is more visible than most vertical fractures cases.

Since the late 1980s, radiographic films have been replaced with digital radiographic systems. Manufacturers offer two types of digital image receptors-photostimulable phosphor plates, and sensors-which have different 
technical characteristics (e.g., physical constitution, and spatial resolution) that can cause distinct image quality $[5,6]$. Both types of systems have numerous advantages over films, such as reduced radiation dose delivered to the patient, the possibility of image storage in digital media, and fast image display, transmission, and communication between professionals and between professionals and patients [7].

Nevertheless, the quantity and size of the image files can overload the digital storage media, hampering their transmission. A way to overcome this limitation and improve clinical workflow is to compress the radiographic images after their acquisition and to export them from the native software into different file formats [7,8]. Although several storage media platforms have become available, a reduction in the file size may be advantageous to avoid waste of virtual space, time, and money. It is possible to export the radiographic images into various file formats, such as tagged image file format (TIFF), bitmap-windows pattern (BMP), and portable network graphics (PNG), which represent a lossless (reversible) compression, in which all image data is preserved. On the other hand, some image file formats, such as joint photographic experts group (JPEG), involve a lossy (irreversible) compression, with loss of image data [7]. The major difference among these types of file formats is the level of compression employed, with TIFF presenting the lowest level of compression, and BMP, PNG, and JPEG formats having higher compression levels [7-9].

Depending on the file format in which it is exported, the same radiographic image can have different file sizes. The compression ratio (CR) determines the reduction in the image file between the original and compressed images, and is expressed as absolute or percentage values [8]. CR values between 1:2 and 1:4 represent no loss of graphic information, while $\mathrm{CR}$ values up to 1:40 are related to loss of graphic information on the compressed image $[8,10]$. Therefore, compression of image files should be seen as an advantage in clinical practice only if the diagnostic accuracy is not negatively affected.

Previous studies have reported the influence of the radiograph file format (i.e., the compression level) on the detection of caries lesions, periapical lesions, root resorptions, and root fractures in different radiography modalities (periapical, panoramic, and cephalometric radiography) [7-15]. However, the cited study on root fractures included both horizontal and vertical root fractures in the assessed sample, without specifying the influence of the file format on each type of fracture individually. Also, the only type of image compression evaluated was JPEG [11]. Therefore, due to the challenge of identifying VRF and the variety of image compression options, the aim in this study was to evaluate the influence of the file format of digital periapical radiographs on the diagnosis of VRFs acquired with different digital systems.

\section{Materials and methods}

\section{Study design}

The present study had an in-vitro design and was conducted after acceptance by the local Institutional Review Board (IRB) (protocol number \#4.231.578).

\section{Sample selection and preparation}

The sample was composed of 34 single-rooted human teeth, including lower incisors, canines and premolars and divided into the control group (without VRF; $n=15$ teeth) and the experimental group (with VRF; $n=19$ teeth). All teeth were collected from a private dental clinic after being extracted mainly due to the presence of periodontal disease, extensive coronal caries lesions or orthodontic indication. All teeth were disinfected in $2 \%$ glutaraldehyde and submitted to clinical and radiographic evaluation to ensure the absence of dental anomalies, calcification, endodontic treatment, open apex, resorptive lesions, and fractures. All teeth were sectioned at the level of the cementoenamel junction to avoid memorization of tooth morphology and bias related to coronary fracture identification during the evaluation. Then, vertical fractures were induced in each of 19 roots (experimental group), using a universal testing machine (INSTRON 4411, Instron Corporation, Canton, MA) and following a previously established methodology [3]. Transillumination using a LED light (Ultralume 5, Ultradent Products Inc., South Jordan, UT, USA) was used to verify the presence of the VRFs, which were located at least at the cervical and middle third of the root.

\section{Image acquisition and export}

The roots were individually included in alveolar sockets of a sectioned human mandible for periapical radiography acquisition. Also, to simulate a clinical scenario, a tongue made of wax was placed behind the image receptor [1].

With the aid of a custom-made acrylic apparatus, the radiographic images were acquired following the parallelism technique. This apparatus was composed of a locator ring to standardize the position of the position-indicating device of the X-ray unit (distance focus-receptor standardized in $40 \mathrm{~cm}$, horizontal angulation of $90^{\circ}$, and vertical angulation of $0^{\circ}$ ). Further, the apparatus allowed to maintain the distance between the object and the receptor in $1.5 \mathrm{~cm}$. To reproduce a clinical scenario and mimic the 
attenuation of the X-rays by the soft tissues, an acrylic block of $2.5 \mathrm{~cm}$ in thickness was settled in front of the buccal cortical plate of the mandible.

The periapical radiographic images were acquired using two radiographic systems with distinct technologies:

1. Phosphor plate (PSP): Digora Optime (Soredex, Tuusula, Finland), size 2, 8-bit contrast resolution, and theoretical spatial resolution of $14.3 \mathrm{lp} \mathrm{mm}^{-1}$.

2. CMOS sensor: Digora Toto-Scanora (Soredex, Tuusula, Finland), size 2, 12-bit contrast resolution, and theoretical spatial resolution of $26.3 \mathrm{lp} \mathrm{mm}^{-1}$.

All images were obtained using the same X-ray unit (FocusTM-Instrumentarium Dental Inc., Milwaukee, WI, USA), under the same acquisition protocol: $60 \mathrm{kVp}, 7 \mathrm{~mA}$, exposure time as recommended by the receptors' manufacturers ( $0.3 \mathrm{~s}$ for Digora Optime, and $0.18 \mathrm{~s}$ for Digora Toto), and standardized focus-receptor distance of $40 \mathrm{~cm}$.
After radiographic acquisition, the images were individually exported from their native software into the four different file formats-TIFF, BMP, PNG, and JPEG-, totaling 272 radiographs (34 radiographs $\times 2$ digital systems $\times 4$ file formats). The Table 1 shows the average file size of each radiographic image for both systems studied, according to the experimental conditions of the present study. Also, Figs. 1 and 2 show radiographs of a sound and of a fractured root, respectively, obtained with the two digital systems tested and exported into the four different file formats.

\section{Image assessment}

Images were independently evaluated by five oral and maxillofacial radiologists with five years of experience in radiographic diagnosis, who were blinded concerning the experimental design, in a medical display (MDRC2124 , Barco N.V., Courtray, Belgium) with $1920 \times 1200$ pixels resolution. The evaluations were conducted using
Table 1 Mean and standard deviation values of the file sizes (in kilobyte), compression percentage, and compression ratio according to the radiographics images and file formats evaluated in the two tested digital systems

\begin{tabular}{|c|c|c|c|c|c|}
\hline \multirow[t]{2}{*}{ Digital systems } & & \multicolumn{4}{|c|}{ File formats } \\
\hline & & TIFF & BMP & PNG & JPEG \\
\hline \multirow[t]{3}{*}{ Digora Optime } & File size (standard deviation) & $\begin{array}{l}2156 \\
(5.19)\end{array}$ & $\begin{array}{l}2152 \\
(4.89)\end{array}$ & $\begin{array}{l}411 \\
\quad(25.90)\end{array}$ & $\begin{array}{l}106 \\
(8.21)\end{array}$ \\
\hline & Compression percentage ${ }^{a}$ & - & 0 & 81 & 95 \\
\hline & Compression ratio ${ }^{\mathrm{a}}$ & - & $1: 1$ & $1: 5$ & $1: 20$ \\
\hline \multirow[t]{4}{*}{ Digora Toto } & File size & 190 & 1900 & 320 & 74 \\
\hline & Standard deviation & 70.00 & 0.00 & 20.00 & 3.60 \\
\hline & Compression percentage $^{a}$ & - & 0 & 83 & 96 \\
\hline & Compression ratio ${ }^{\mathrm{a}}$ & - & $1: 1$ & $1: 6$ & $1: 26$ \\
\hline
\end{tabular}

${ }^{\mathrm{a}}$ Comparing with TIFF file format
Fig. 1 Radiographic images of the control group (without vertical root fracture) in different image file formats, for the two intraoral digital systems

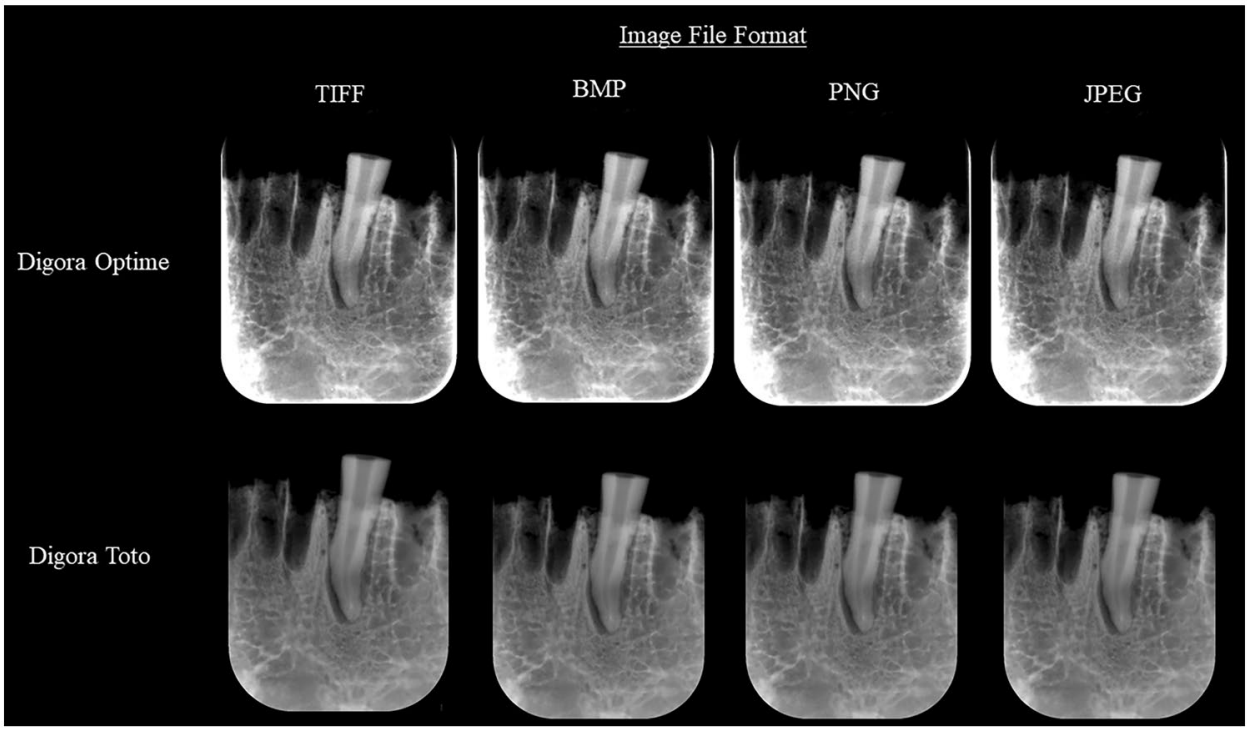


Fig. 2 Radiographic images of the experimental group (with vertical root fracture) in different image file formats, for the two intraoral digital systems

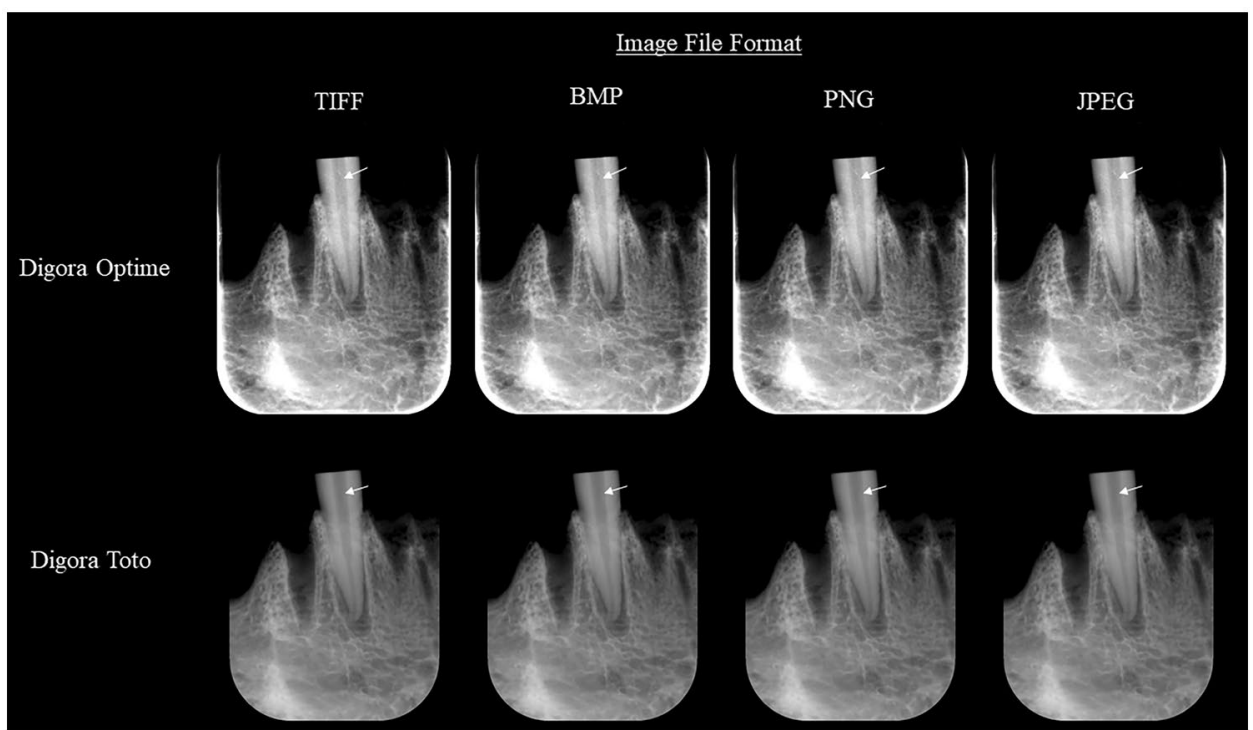

the JPEGView image viewer. Before the evaluations, the examiners were instructed about the assessment of VRFs, using periapical radiographs that were not included in the sample as examples. To simulating a clinical scenario, they were also allowed to adjust image brightness, contrast, and zoom settings. Then, examiners should classify each image according to the presence or absence of VRF, using a 5-point scale: 1 -definitely absent; 2 -probably absent; 3-uncertain; 4-probably present; 5-definitely present. To reduce the possibility of image memorization and prevent visual weariness, the evaluators were oriented to assess a maximum of 20 images per day. Thirty days after the analysis was concluded, $30 \%$ of the sample was re-evaluated to calculate the intra-examiner agreement.

All evaluators were blind for the image file format, as all images were codified and randomized to prevent identification.

\section{Statistical analysis}

Data were analyzed in the Statistical Package for Social Sciences software v. 24.0 (IBM Corp., Armonk, NY, USA). Intra- and inter-examiner agreements regarding the diagnosis of VRF were calculated by weighted Kappa test. Diagnostic values [area under the receiver operating characteristic (ROC) curve, specificity, and sensitivity] for the diagnosis of VRF were calculated. The data obtained were individually compared by two-way analysis of variance with Tukey's test as a post-hoc, considering the file formats and radiographic systems as the studied factors. A significance level of 5\% was adopted for all analyses.
Table 2 Intra- and inter-examiner agreements for the diagnosis of vertical root fractures

\begin{tabular}{llllll}
\hline Observer & 1 & 2 & 3 & 4 & 5 \\
\hline 1 & 0.57 & 0.58 & 0.52 & 0.29 & 0.57 \\
2 & & 0.71 & 0.49 & 0.29 & 0.51 \\
3 & & & 0.73 & 0.36 & 0.60 \\
4 & & & & 0.56 & 0.35 \\
5 & & & & & 0.81 \\
\hline
\end{tabular}

\section{Results}

As showed in Table 2, the values of intra-examiner agreement varied from moderate (0.56) to almost perfect $(0.81)$, while the values of inter-examiner agreement varied from fair (0.29) to moderate (0.60), according to the Landis and Koch scale [16].

The diagnostic values (area under the ROC curve, sensitivity, and specificity) of VRF according to the different image file formats (TIFF, BMP, PNG and JPEG) and digital radiographic systems (Digora Toto and Digora Optime) are shown in Table 3. The image file format did not influence the diagnostic values for any of the systems tested $(p>0.05)$. Nonetheless, Nonetheless, the digital radiographic systems type influenced the values of the area under the ROC curve $(p=0.001)$; the CMOS sensor Digora Toto demonstrated greater values of area under the ROC curve (values ranging from 0.90 to 0.93 ) than those of the PSP receptor Digora Optime for the detection of VRF (values ranging from 0.77 to 0.82 ) for all 
Table 3 Average (SD) of the AUC, sensitivity and specificity values for the diagnosis of vertical root fracture according to the conditions assessed
Fig. 3 ROC curves comparing different image file formats (TIFF, BMP, PNG, and JPEG) within each digital radiographic system (Digora Toto and Digora Optime)

\begin{tabular}{|c|c|c|c|c|c|c|}
\hline \multirow[t]{2}{*}{ File format } & \multicolumn{2}{|l|}{ AUC } & \multicolumn{2}{|l|}{ Sensitivity } & \multicolumn{2}{|l|}{ Specificity } \\
\hline & $\begin{array}{l}\text { Digora } \\
\text { Toto }\end{array}$ & $\begin{array}{l}\text { Digora } \\
\text { Optime }\end{array}$ & $\begin{array}{l}\text { Digora } \\
\text { Toto }\end{array}$ & $\begin{array}{l}\text { Digora } \\
\text { Optime }\end{array}$ & $\begin{array}{l}\text { Digora } \\
\text { Toto }\end{array}$ & $\begin{array}{l}\text { Digora } \\
\text { Optime }\end{array}$ \\
\hline BMP & $0.91(0.03)$ & $0.82(0.10)$ & $0.80(0.08)$ & $0.82(0.11)$ & $0.87(0.12)$ & $0.67(0.36)$ \\
\hline JPEG & $0.90(0.05)$ & $0.82(0.10)$ & $0.81(0.10)$ & $0.78(0.12)$ & $0.80(0.25)$ & $0.75(0.37)$ \\
\hline PNG & $0.90(0.03)$ & $0.77(0.17)$ & $0.81(0.06)$ & $0.84(0.12)$ & $0.88(0.17)$ & $0.65(0.33)$ \\
\hline TIFF & $0.93(0.02)$ & $0.79(0.10)$ & $0.83(0.09)$ & $0.78(0.16)$ & $0.88(0.14)$ & $0.67(0.32)$ \\
\hline$p$ system & 0.001* & & 0.809 & & 0.054 & \\
\hline$p$ format & 0.929 & & 0.924 & & 1.000 & \\
\hline$p$ interactions & 0.817 & & 0.779 & & 0.884 & \\
\hline
\end{tabular}

$A U C$ area under the receiver operating curve, $S D$ standard deviation

*Indicate significant difference $(p<0.05)$ between the radiographic systems, for all file formats

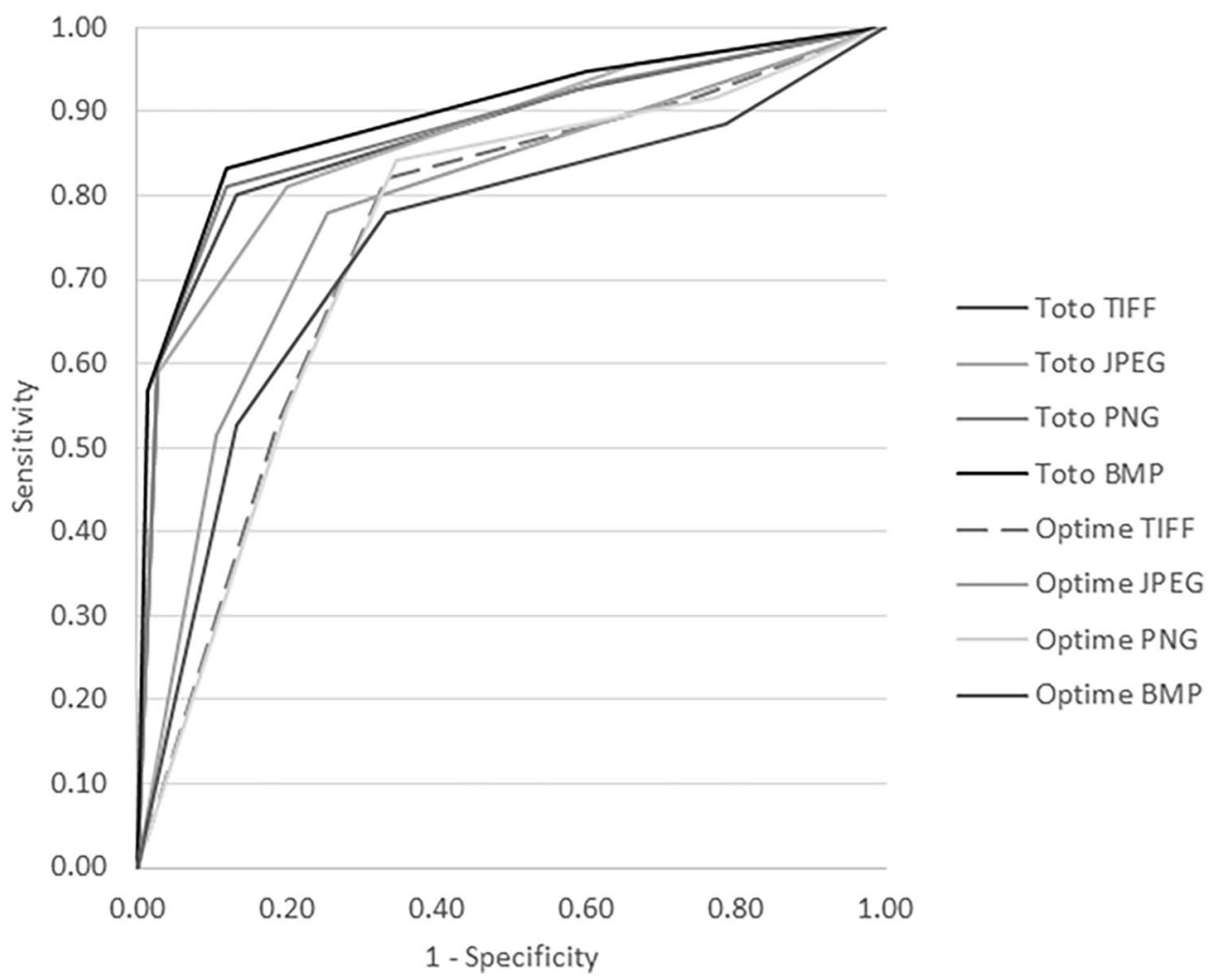

image file formats. The graph representing the ROC curves illustrates their similarity when comparing different image file formats within each digital radiographic system; it is also possible to observe the difference between the digital radiographic systems (Fig. 3). No significant difference was observed between digital systems regarding the values of sensitivity $(p=0.81)$ and specificity $(p=0.05)$.

\section{Discussion}

The initial hypothesis that file formats with a higher degree of compression would negatively influence the VRF diagnosis was refuted by our results. Our findings demonstrated no differences in any of the diagnostic values for the detection of VRF among TIFF, BMP, PNG, and JPEG formats.

The findings of the present study are in accordance with the results of previous investigations that compared the diagnostic values obtained with different file formats and compression levels for various diagnostic tasks in dentistry $[7,11,12,15,17,18]$. No differences have been found between TIFF and two different levels of compressed JPEG images in the diagnosis of root fractures [10]. Gegler et al. [15] did not find differences among TIFF, BMP, and JPEG file formats in a digital subtraction technique for the diagnosis of simulated external root resorptions. Likewise, in addition to the above-mentioned formats, a recent study also evaluated 
the PNG format in the diagnosis of simulated internal and external root resorptions on periapical radiographs and found no significant differences among the different image file formats assessed [8]. It is important to point out that a significant difference between different levels of JPEG compression was found in a subjective analysis for caries lesions detection; however, the compression ratio used was 1:48, which is an excessive and unusual level of compression, which may significantly impair image quality [9]. Madlum et al. [7] evaluated the diagnosis of proximal incipient caries diagnoses in different image file formats, with the JPEG format at a compression ratio of 1:27, and even then, no significant difference was found between the formats. For the diagnosis of vertical root fractures, to our knowledge, only the TIFF and JPEG formats have been previously analyzed [11].

Although the diagnostic values of sensitivity and specificity did not differ between the radiographic systems tested (Digora Optime, and Digora Toto) in our study, the accuracy values of the CMOS sensor were significantly greater than those of the PSP in the diagnosis of VRF, for all image file formats. Greater accuracy of Digora Toto in comparison with Digora Optime was also reported by previous studies that assessed VRFs, and horizontal root fractures $[1,5]$. Such findings may be related to the greater spatial resolution provided by Digora Toto $\left(26.3 \mathrm{lp} \mathrm{mm}^{-1}\right)$, in comparison with Digora Optime (14.3 $\left.\mathrm{lp} \mathrm{mm}^{-1}\right)$. This concept is associated with the ability to display physical separation between two images [5]. Another point to be taken into consideration is the contrast resolution. In the present study, 8-bit and 12-bit contrast resolutions were used as a consequence of the sensor and phosphor plate-based systems used. Technically, a greater bit depth allows a better representation of structures with discrete differences in their composition and physical aspects. Heo et al. [19] found that 12-bit images were superior to 8-bit images in the detection of subtle radiographic contrast, corroborating our findings. On the other hand, Wenzel et al. [20] showed that the detection of small caries lesions was not different between 8-, 12-, and 16-bit images. Despite this, the authors believe that the most important evaluation of the present study, which was the comparison among different file formats in the detection of vertical root fractures, was not affected by the bit depth of the systems employed.

According to the consulted literature, only one previous research has assessed the effect of image compression on the detection of root fractures. This previous study concluded that the file format does not affect such diagnosis [10]. Nevertheless, it is not possible to assure the absence of such influence on the diagnosis of VRF, since the authors evaluated both horizontal and vertical root fractures in the same sample, not discriminating specific results for each type of root fracture.
The methodology used in the current investigation, based on the experience level of the observers and their interpretations, strongly supports previous conclusions that have indicated no differences among different image compression levels and file formats in the detection of root fractures. Furthermore, this study considered multiple factors that could affect the results, such as different radiographic systems and file formats, which prevents the underestimation or overestimation of the accuracy values $[5,10]$.

Moreover, the findings of the present study may be considered especially important in this pandemic period, since the digital transmission of radiographic files has become even more important than in the short past [21]. The online transmission of radiographs facilitates communication between professionals, and between professionals and patients, reduces paper waste, in addition to being an alternative to minimizing the risk of transmission of COVID-19 through printed radiographs [7,22]. Given that and based on our results, it seems to be advisable for professionals to export radiographic images acquired for VRF diagnosis into JPEG file format, i.e., the highest degree of compression, which requires less space for storage and facilitates the online transmission, but it is important to consider local regulations before clinical application. However, our results also support that professionals can use the format that best fits their convenience without compromising the diagnosis.

\section{Conclusion}

The file format of periapical radiographs does not influence the diagnosis of VRF regardless of the digital radiography system.

Acknowledgements This study was financed in part by the Coordenação de Aperfeiçoamento de Pessoal de Nível Superior-Brasil (CAPES)_Finance Code 001.

Author contributions MM-V conceptualization, investigation, writing and revising the draft critically for important intellectual content, final approval of the version to be published and agreement to be accountable for all aspects of the work. RCF co-author; conceptualization, investigation, formal analysis, and interpretation of data for the work, writing and revising the draft critically for important intellectual content and agreement to be accountable for all aspects of the work. AFG co-author; methodology, formal analysis, and interpretation of data for the work, revising the draft critically for important intellectual content and agreement to be accountable for all aspects of the work. FN-R co-author; methodology, revising the draft critically for important intellectual content and agreement to be accountable for all aspects of the work. YN co-author; methodology, revising the draft critically for important intellectual content, final approval of the version to be published and agreement to be accountable for all aspects of the work. MLO co-author; revising the draft critically for important intellectual content, final approval of the version to be published and agreement 
to be accountable for all aspects of the work. DQF supervisor; conceptualization, investigation, revising the draft critically for important intellectual content, final approval of the version to be published and agreement to be accountable for all aspects of the work.

Funding This study was financed in part by the Coordenação de Aperfeiçoamento de Pessoal de Nível Superior-Brasil (CAPES)—Finance Code 001.

\section{Declarations}

Conflict of interest The authors declare that they have no conflict of interest.

Ethical approval This study was carried out after local Institutional Review Board approval (protocol number \#4.231.578). All procedures followed were in accordance with the ethical standards of the responsible committee on human experimentation (institutional and national) and with the Helsinki Declaration of 1975, as revised in 2008 (5). Informed consent was obtained from all patients for being included in the study.

Informed consent Not applicable

\section{References}

1. Farias Gomes A, Nejaim Y, Fontenele RC, Haiter-Neto F, Freitas DQ. Influence of the incorporation of a lead foil to intraoral digital receptors on the image quality and root fracture diagnosis. Dentomaxillofac Radiol. 2019;48:1-7. https://doi.org/10.1259/dmfr. 20180369.

2. Uysal S, Akcicek G, Yalcin ED, Tuncel B, Dural S. The influence of voxel size and artifact reduction on the detection of vertical root fracture in endodontically treated teeth. Acta Odontol Scand. 2021;79:354-8. https://doi.org/10.1080/00016357.2020.1859611.

3. Vasconcelos TV, Santaella GM, Nascimento HA, Rovaris K, Ambrosano GM, Freitas DQ. Digital radiographs displayed on different devices: effect on the detection of vertical root fractures. Int Endod J. 2016;49:386-92. https://doi.org/10.1111/iej.12466.

4. Nascimento HAR, Neves FS, Azevedo-Vaz SL, Duque TM, Ambrosano GMB, Freitas DQ. Impact of root fillings and posts on the diagnostic ability of three intra-oral digital radiographic systems in detecting vertical root fractures. Int Endod J. 2015;48:864-71. https://doi.org/10.1111/iej.12382.

5. Nejaim Y, Gomes AF, Silva EJ, Groppo FC, Haiter NF. The influence of number of line pairs in digital intra-oral radiography on the detection accuracy of horizontal root fractures. Dent Traumatol. 2016;32:180-4. https://doi.org/10.1111/edt.12243.

6. Fontenele RC, Nejaim Y, Farias Gomes A, Gaêta-Araujo H, Haiter-Neto F, Freitas DQ. Does the addition of a lead foil to digital radiographic receptors influence image contrast and approximal caries lesions diagnosis? Dentomaxillofac Radiol. 2020;49:1-6. https://doi.org/10.1259/dmfr.20190384.

7. Madlum DV, Gaêta-Araujo H, Brasil DM, Lima CAS, Oliveira ML, Haiter-Neto F. Influence of the file format and transmission app on the radiographic diagnosis of caries lesions. Oral Surg Oral Med Oral Pathol Oral Radiol. 2020. https://doi.org/10.1016/j. oooo.2020.11.013

8. Miranda-Viana M, Madlum DV, Oliveira-Santos N, Gaêta-Araujo $\mathrm{H}$, Haiter-Neto F, Oliveira ML. Influence of the image file format of digital periapical radiographs on the diagnosis of external and internal root resorptions. Clin Oral Investig. 2021. https://doi.org/ 10.1007/s00784-021-03803-0.

9. Wenzel A, Moystad A. Work flow with digital intraoral radiography: a systematic review. Acta Odontol Scand. 2010;68:106-14 https://doi.org/10.3109/00016350903514426.

10. Fidler A, Likar B, Skaleric U. Lossy JPEG compression: easy to compress, hard to compare. Dentomaxillofac Radiol. 2006;35:6773. https://doi.org/10.1259/dmfr/66171128.

11. Noujeim M, Geha H, Shintaku W, Bechara B, Kashi KA Effect of JPEG compression on the diagnostic accuracy of periapical images in the detection of root fracture. Dent Traumatol. 2012;28:233-7. https://doi.org/10.1111/j.1600-9657.2011. 01076.x.

12. Janhom A, van der Stelt PF, van Ginkel FC. Interaction between noise and file compression and its effect on the recognition of caries in digital imaging. Dentomaxillofac Radiol. 2000;29:20-7. https://doi.org/10.1038/sj/dmfr/4600488.

13. Provedel LF, Pimentel BN, Jesus TM, Lacerda AP, Rubira-Bullen IR, Sarmento VA. Effects of image compression on linear measurements of digital panoramic radiographs. Braz Dent J. 2016;27:757-60. https://doi.org/10.1590/0103-6440201601157.

14. Duarte H, Vieck R, Siqueira DF, Angelieri F, Bommarito S, Dalben G, Sannomiya EK. Effect of image compression of digital lateral cephalograms on the reproducibility of cephalometric points. Dentomaxillofac Radiol. 2009;38:393-400. https://doi. org/10.1259/dmfr/40996636.

15. Gegler A, Mahl C, Fontanella V. Reproducibility of and file format effect on digital subtraction radiography of simulated external root resorptions. Dentomaxillofac Radiol. 2006;35:10-3. https://doi. org/10.1259/dmfr/86879455.

16. Landis JR, Koch GG. The measurement of observer agreement for categorical data. Biometrics. 1977;33:159-74. https://doi.org/10. 2307/2529310.

17. Koenig L, Parks E, Analoui M, Eckert G. The impact of image compression on diagnostic quality of digital images for detection of chemically-induced periapical lesions. Dentomaxillofac Radiol. 2004;33:37-43. https://doi.org/10.1259/dmfr/30099843.

18. Pabla T, Ludlow JB, Tyndall DA, Platin E, Abreu M Jr. Effect of data compression on proximal caries detection: observer performance with DenOptix photostimulable phosphor images. Dentomaxillofac Radiol. 2006;32:45-9. https://doi.org/10.1259/dmfr/ 19650275.

19. Heo MS, Choi DH, Benavides E, Huh KH, Yi WJ, Lee SS, Choi $\mathrm{SC}$. Effect of bit depth and $\mathrm{kVp}$ of digital radiography for detection of subtle differences. Oral Surg Oral Med Oral Pathol Oral Radiol Endod. 2009;108:278-83. https://doi.org/10.1016/j.tripl eo.2008.12.053.

20. Wenzel A, Haiter-Neto F, Gotfredsen E. Influence of spatial resolution and bit depth on detection of small caries lesions with digital receptors. Oral Surg Oral Med Oral Pathol Oral Radiol Endod. 2007;103:418-22. https://doi.org/10.1016/j.tripleo.2006.05.016.

21. Rosado LP, Crusoé-Rebello I, Oliveira ML, Freitas DQ, Neves FS. Dental teleradiology: a powerful strategy to overcome the impact of COVID-19. Acad Radiol. 2020;10:1492-3. https://doi.org/10. 1016/j.acra.2020.07.034

22. Fontenele RC, Gomes AF, Freitas DQ. Oral radiology practice in dental schools during the COVID-19 pandemic: what will be the new normal? Imaging Sci Dent. 2020;50:265-7. https://doi.org/ 10.5624/isd.2020.50.3.265.

Publisher's Note Springer Nature remains neutral with regard to jurisdictional claims in published maps and institutional affiliations. 